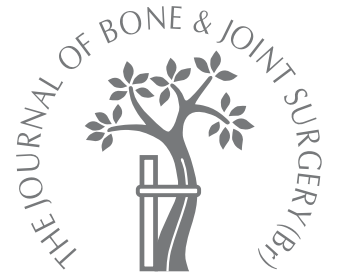

A. J. Anderson, G. M. Towns, N. Chiverton

From Northern General Hospital, Sheffield, England

A. J. Anderson, FRCS(Tr \& Orth), Orthopaedic Specialist Registrar

N. Chiverton, FRCS(Tr \&

Orth), Consultant Spinal

Surgeon

Department of Spinal Surgery Northern General Hospital,

Herries Road, Sheffield S5 7AU,

UK.

In. M. Towns, FRCS(Ed), Consultant Neurological and Spinal Surgeon

Department of Neurosurgery Leeds General Infirmary, Great George Street, Leeds LS1 3EX UK.

Correspondence should be sent to $\mathrm{Mr}$ A. J. Anderson at 35

Strathtay Road, Eccleshall,

Sheffield S11 7GU, UK; e-mail:

alexander-

anderson@btinternet.com

(C2006 British Editorial Society of Bone and Joint Surgery doi:10.1302/0301-620X.88B11. $18082 \$ 2.00$

$J$ Bone Joint Surg $[\mathrm{Br}]$ 2006;88-B:1464-8.

Received 3 May 2006; Accepted 26 July 2006

\title{
Traumatic occipitocervical disruption: a new technique for stabilisation
}

\author{
CASE REPORT AND LITERATURE REVIEW
}

Traumatic atlanto-occipital dislocation in adults is usually fatal and survival without neurological deficit is rare. The surgical management of those who do survive is difficult and controversial. Most authorities recommend posterior occipitoaxial fusion, but this compromises cervical rotation. We describe a case in which a patient with a traumatic atlanto-occipital disruption but no neurological deficit was treated by atlanto-occipital fusion using a new technique consisting of cancellous bone autografting supported by an occipital plate linked by rods to lateral mass screws in the atlas. The technique is described in detail. At one year the neck was stable, radiological fusion had been achieved, and atlantoaxial rotation preserved.

The rationale behind this approach is discussed and the relevant literature reviewed. We recommend the technique for injuries of this type.

Traumatic atlanto-occipital dislocation is usually fatal. Fewer than 100 survivors have been reported. However, with improving emergency care the number of patients who survive is increasing. ${ }^{1}$ The injury is predominantly ligamentous and occurs as a result of rapid deceleration of the trunk in relation to the head.

The management is controversial and the surgeon faces a number of technical challenges. Most authors advocate occipitocervical fusion, for which various techniques have been described. ${ }^{2-5}$ These invariably extend down to the axis, resulting in a significant loss of cervical rotation and possible increase in the stresses on the adjacent motion segments. ${ }^{6}$ In our opinion, this loss of rotation is unacceptable in cases where there is no neurological deficit. Two studies have previously described successful techniques for atlanto-occipital fusion. ${ }^{6,7}$ Sponseller and Cass $^{7}$ treated paediatric injuries with wire fixation, which is known to be less rigid than a screw, plate and rod construct. ${ }^{2,4} \mathrm{Grob}^{6}$ used transarticular atlanto-occipital screws, but felt that additional atlantoaxial screws were needed to achieve adequate stability. ${ }^{1}$

We describe a technique of posterior atlanto-occipital instrumentation with placement of polyaxial screws in the pedicle of the atlas (C1), an occipital T-plate and rods.

\section{Case report}

A 54-year-old man was admitted to a local District General Hospital after a motorcycle accident. He had been thrown off while travelling at $45 \mathrm{mph}$ and had slid down a grassy bank into some bushes. He was treated according to advanced trauma and life support (ATLS) principles from the point of extraction at the accident scene. On arrival in the Accident and Emergency Department he was complaining of pain in his right shoulder and neck, but despite his describing paralysis in all four limbs for several minutes immediately after the accident, no abnormal neurological findings were identified. After primary and secondary surveys, radiographs revealed a displaced fracture of the midshaft of the right clavicle and massive soft-tissue swelling in front of the upper cervical vertebrae (Fig. 1).

An MR scan (Fig. 2) confirmed a pre-vertebral haematoma and showed disruption of the anterior longitudinal ligament (atlanto-occipital membrane) and posterior longitudinal ligament (tectorial membrane) consistent with complete atlanto-occipital ligamentous disruption. He was kept in a stiff collar and transferred to the regional spinal surgery unit for further management. There, a CT scan revealed, in addition, a small avulsion fracture of the right occipital condyle (Fig. 3). Operative stabilisation was felt to be appropriate. 


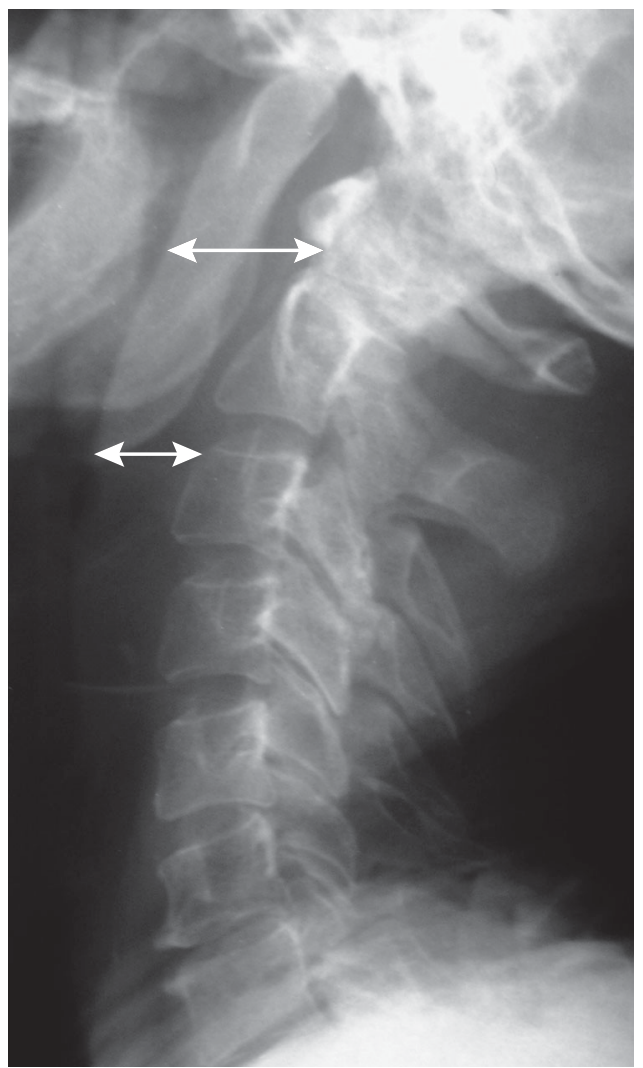

Fig. 1

Lateral radiograph of the cervical spine. Note the prevertebral swelling (arrows).

Atlanto-occipital posterior fusion technique. Under general anaesthetic the patient was placed prone on an operating table. The cervical spine was kept in alignment with a Mayfield clamp (OMI Surgical Products, Cincinnati, Ohio) and the position checked with an image intensifier. The posterior arch of the atlas and the occiput up to the superior nuchal line were exposed in the standard manner. Two polyaxial pedicle screws (Summit DePuy Spine; Johnson and Johnson, Leeds, United Kingdom) $26 \mathrm{~mm} \times 3.5 \mathrm{~mm}$ in diameter, were inserted into the atlas using a technique first described by Harms and Melcher ${ }^{8}$ for atlantoaxial instability. The atlantoaxial joint was exposed and the ganglion at C2 retracted caudally so that the entry point for the screw could be accurately defined. This lies at the midpoint of the junction of the arch of the atlas with the middle of the posteroinferior part of its lateral mass. The position of the screws was confirmed with an image intensifier. Next, a T-plate was fixed to the occiput with two midline bicortical occipital screws. The polyaxial screws and T-plate were connected with two parallel contoured $3 \mathrm{~mm}$ titanium rods. A cancellous bone graft from the posterior iliac crest was applied to the underlying decorticated bone, taking care not to involve the axis (Fig. 4).

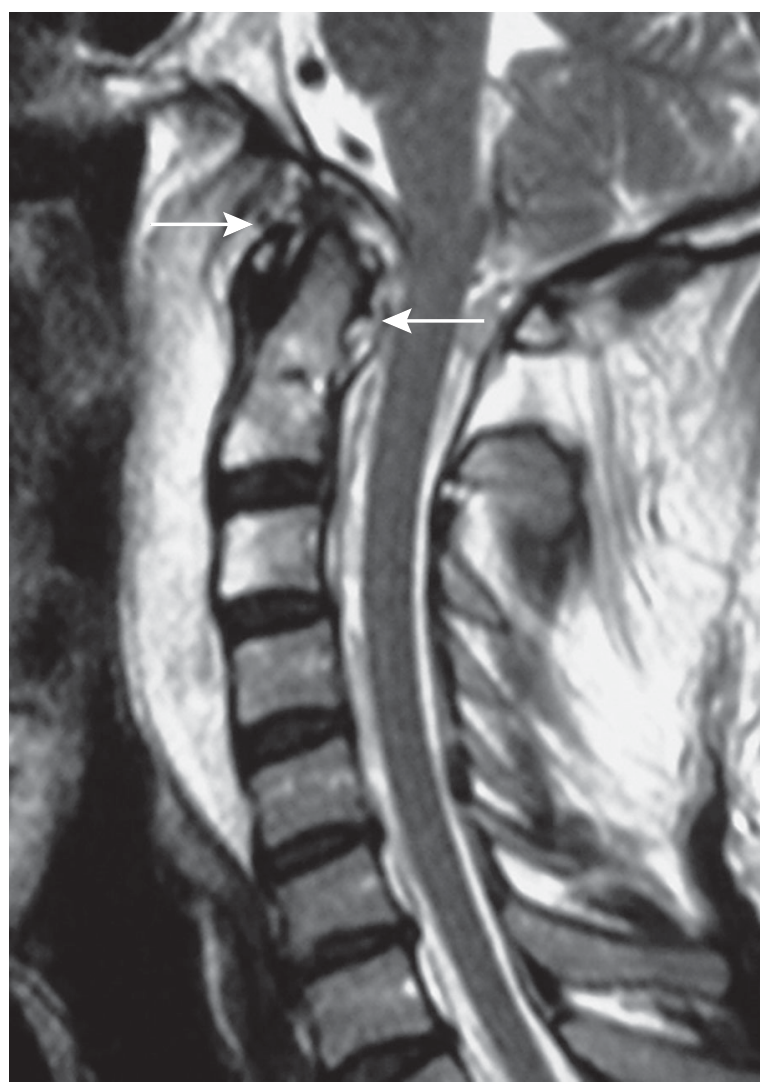

Fig. 2

Cervical MRI showing disruption of the anterior and posterior longitudinal ligaments.

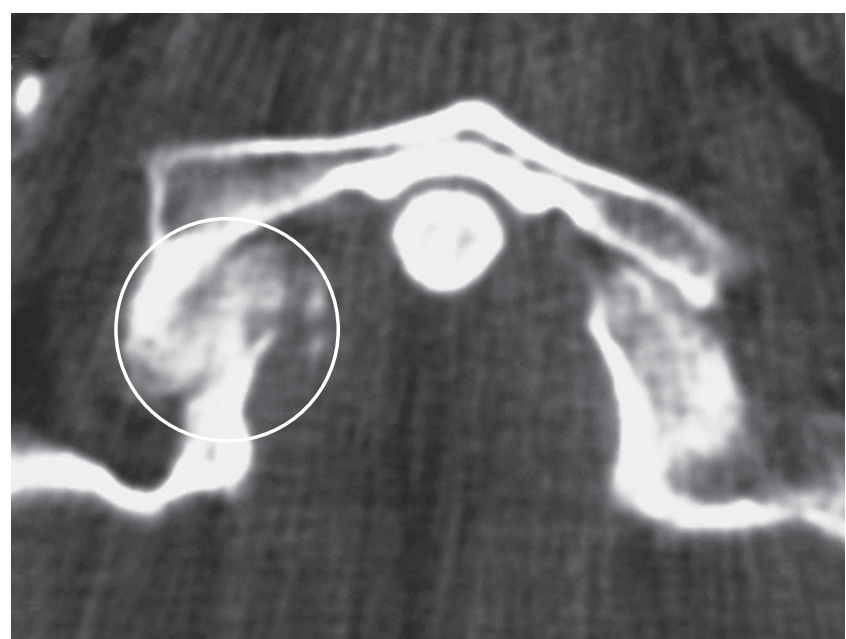

Fig. 3

Axial CT showing a flake avulsion fracture from the right occipital condyle.

There were no peri-operative complications. The patient was immobilised in a Philadelphia collar (Philadelphia cervical collar company, Thorofare, New Jersey) for a further three weeks. 


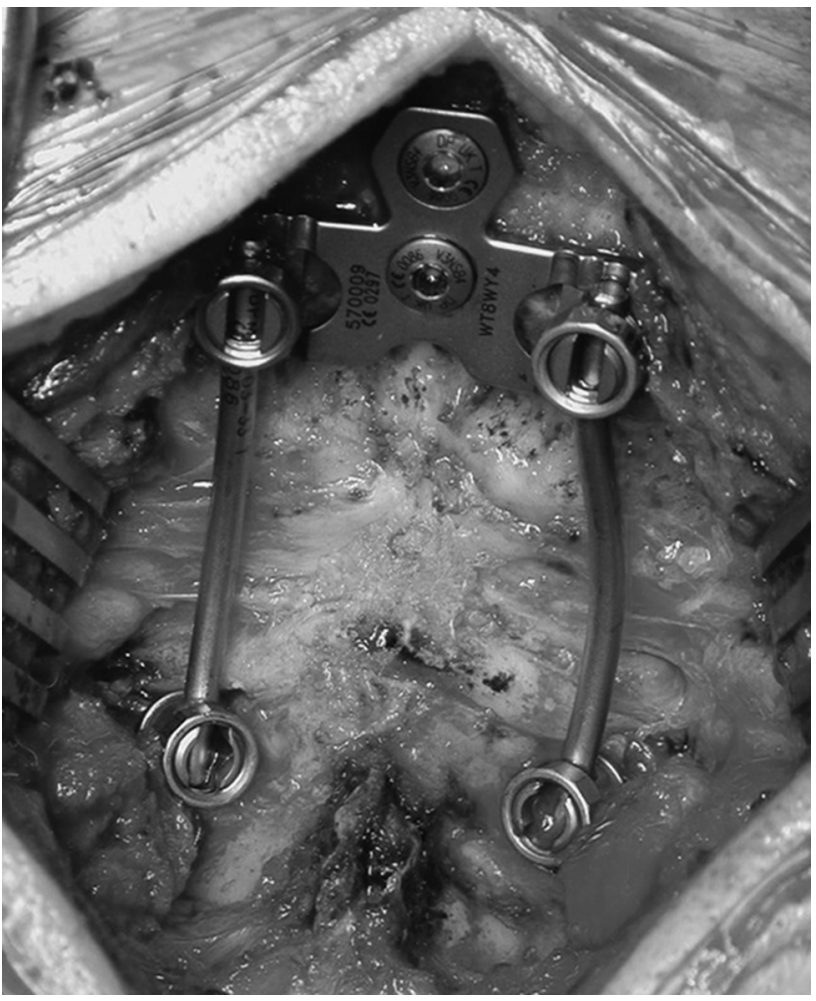

Fig. 4a

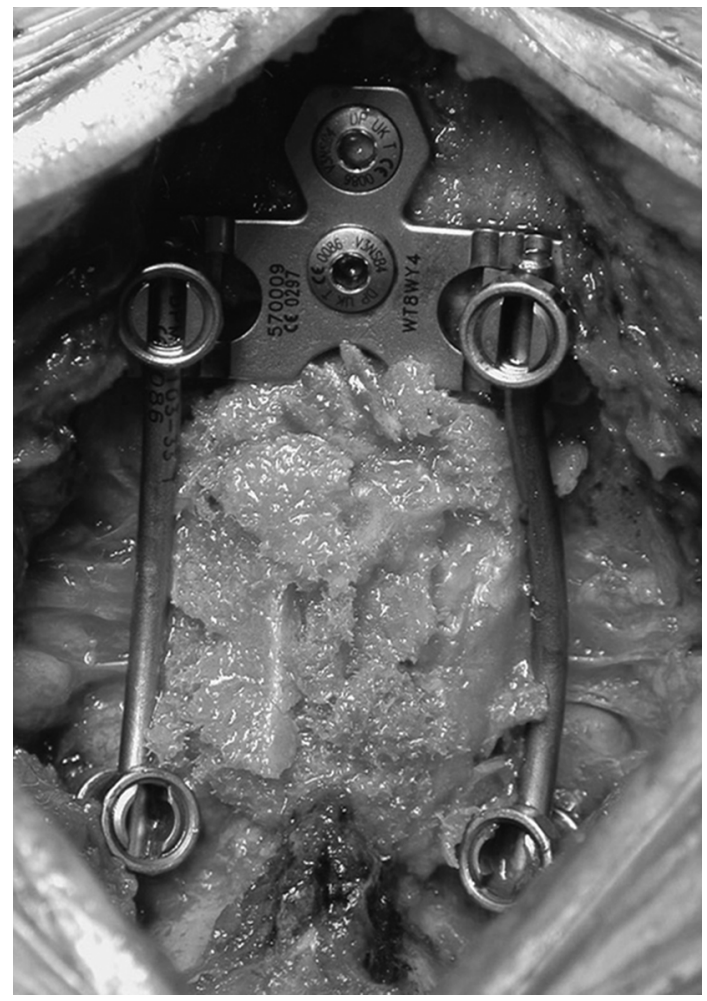

Fig. $4 \mathrm{~b}$

a) The posterior instrumentation and b) the instrumentation with bone graft onlay.
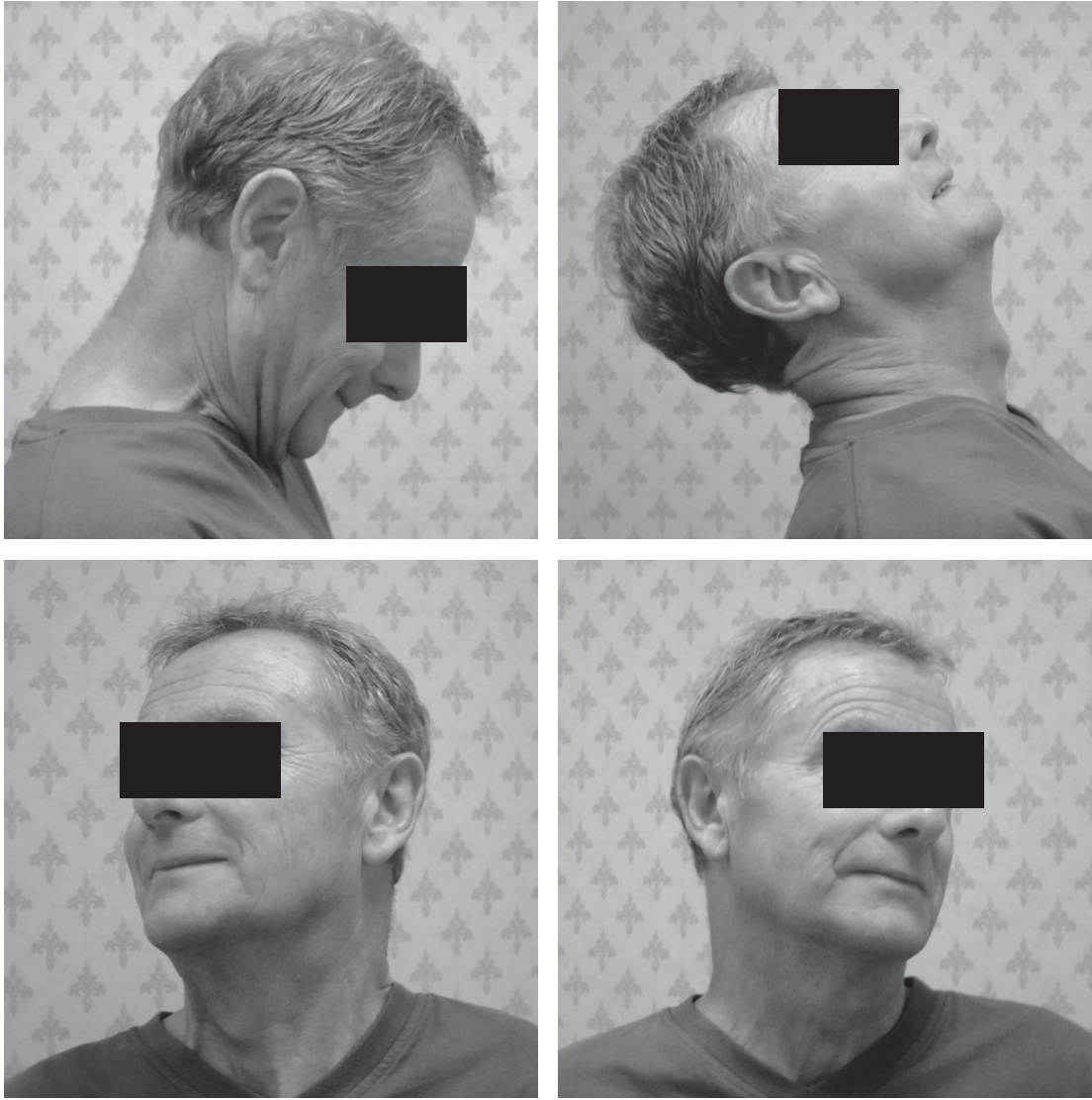

Fig. 5

Range of movement after six months. 


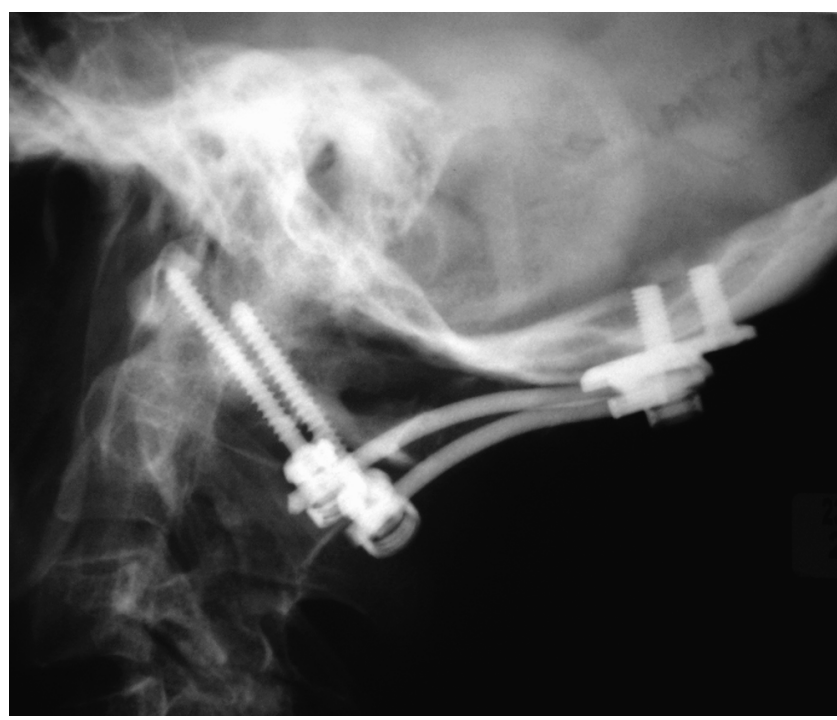

Fig. 6

Lateral radiograph showing fusion at one year.

Six months later he had returned to all normal activities and was symptom free, apart from an occasional occipital headache. His range of movement was excellent, with full flexion and extension, and approximately three-quarters of the normal range of lateral rotation (Fig. 5). There was evidence of bony fusion on lateral radiographs taken after one year (Fig. 6).

\section{Discussion}

The atlanto-occipital joint has little intrinsic bony stability. Its ligaments, the anterior and posterior longitudinal (tectorial), the cruciate (transverse), and the odontoid complex (apical and alar ligaments) confer stability on the joint aided by the joint capsules. ${ }^{9}$ Although it is difficult to interpret the MRI precisely, it appears that all these structures were disrupted in this case (Fig. 2).

The atlanto-occipital joint allows a $13^{\circ}$ combined arc of flexion through to extension. There is little rotation, owing to the attachment of the alar ligaments to the occiput and the axis. These serve as the primary rotatory stabilisers at this level. By contrast, the atlantoaxial joint allows only $10^{\circ}$ of flexion but $65^{\circ}$ of rotation. ${ }^{7}$ In this case, we felt that provided there was no atlantoxial instability, we should attempt to preserve rotation by fusion of the atlanto-occipital motion segment. We also believed that by fusing one level instead of two there would be less stress transferred to the remaining cervical levels.

Traynelis et $\mathrm{al}^{10}$ have classified atlanto-occipital dislocations into three types. type 1 when the occipital condyles lie in front of the atlas; type 2, a pure distraction injury, when the condyles are separated from the atlas without translation, as in this case; and type 3 when the occipital con- dyles lie behind the atlas. Dislocation occurs more commonly in children because in childhood the joint lies in a more horizontal plane and its condyles are smaller. ${ }^{7}$

Anderson and Montesano ${ }^{11}$ have classified fractures of the occipital condyle: a type 1 in which there is an impaction of the condyle, a type II associated with a basilar or skull fracture and a type III resulting from forced rotation and with avulsion of the condyle at its point of attachment to the alar ligament. A type III fracture such as occurred in our case, is often associated with craniovertebral dissociation and is considered unstable.

The clinical presentation of those who survive atlantooccipital dislocation is varied. The clinician should be alert to a history of paresis of the lower cranial nerves, monoparesis, hemiparesis, quadriparesis, respiratory dysfunction or a complete motor deficit of the high cervical cord. ${ }^{1}$ These features may be transient and thus present with severe neck pain but no neurological abnormality.

The diagnosis of atlanto-occipital dislocation can be made on a plain lateral radiograph when there is prevertebral soft-tissue swelling and superior, anterior or posterior translation of the occipital condyles in relation to the atlas. ${ }^{12}$ However, it is frequently missed at initial presentation because of its rarity. ${ }^{1,7,12,13}$ Although we acknowledge that in our case there was no plain radiological evidence of atlanto-occipital dislocation, the history, clinical and MRI findings suggest that there had been a significant distraction force resulting in complete disruption of the atlantooccipital ligaments. Powers et $\mathrm{al}^{14}$ have suggested a standardised approach to aid diagnosis on a plain radiograph using the ratio of the distance between the basion and the posterior arch of the atlas, to the distance from the anterior arch of the atlas to the opisthion. When this ratio is greater than 1, dislocation is likely. Once suspected, both CT and MRI are mandatory to confirm the dislocation, identify any associated fractures, and to assess the extent of ligamentous disruption and spinal cord injury. ${ }^{13}$

The management of atlanto-occipital dislocation aims to restore stability and preserve neurological function. This can be achieved by closed reduction and immobilisation with a cervical orthosis or a halo-body jacket, but great care must be taken when manipulating the neck to avoid further neurological injury. ${ }^{12}$ Although it is possible to manage atlanto-occipital dislocation with external immobilisation, the literature suggests a high incidence of persistent instability and worsening neurological function. ${ }^{1}$ An orthosis can be combined successfully with a posterior fusion, particularly in children, ${ }^{7}$ but because it is biomechanically inferior to internal stabilisation it is not recommended in adults. ${ }^{9}$ Atlanto-occipital dislocation differs slightly from other injuries to the cervical spine in that it is inherently a ligamentous injury. Consequently, most surgeons would advocate operative fusion to achieve long-term stability.

Several methods of stabilising occipitocervical injuries have been described. Anterior approaches are rarely used and only if there is an associated anterior fracture, such as 
a displaced fracture of the dens. ${ }^{9}$ Most are carried out through a posterior approach using a wiring technique or a screw-plate construct. The use of bone graft is essential to achieve fusion. The more stable the construct, the shorter the period of post-operative immobilisation.

Oda et $\mathrm{al}^{4}$ studied the biomechanics of five different occipitocervical fixation constructs in cadaveric spines, and found that screw fixation from the occiput to the pedicle of C2 was the stiffest. Transarticular screws afforded greater stability than hooks and wires, which fared badly. The use of wires also relies on the integrity of the posterior elements and risks injuring the dura when they are passed through occipital burr holes or under the lamina of the spine. The siting of transarticular screws, whether atlanto-occipital ${ }^{6}$ or atlantoaxial, ${ }^{15}$ is also technically demanding, as in $20 \%$ of the population there is anatomical variation in the path of the vertebral artery on one side or the other which precludes placement of a screw. ${ }^{4,8}$ We would expect our construct to function biomechanically in much the same way as Oda's ${ }^{4}$ occipitoaxial pedicle screw fixation, but without the loss of cervical rotation.

Sutterlin et $\mathrm{al}^{2}$ also evaluated three occipito cervical constructs using a cadaveric model. A rectangular rod and wire fixation, a Y-plate, and a custom plate with segmental fixation were used. They demonstrated the biomechanical superiority of a plate and screw construct over a rod-wire technique. Failure of plate and screw constructs occurred in extreme flexion by fracture through the pedicle of the axis. They also showed that an inverted T-plate was significantly stiffer in torsion than an occipital Y-plate.

Currently, the best method of achieving occipital fixation is unknown. The pull-out strength of the screw correlates directly with the thickness of the occipital bone. The midline of the cranium is twice as thick as the lateral part and confers a safe zone between the superior and inferior nuchal lines which avoids the dural venous sinuses. ${ }^{16,17}$ There are, however, understandable concerns about the risk of thrombosis and infection which may occur if these are penetrated.

There is further concern over the risk of irritating the $\mathrm{C} 2$ ganglion with the $\mathrm{C} 1$ lateral mass screw, causing occipital neuralgia. Although this was initially a problem in our case, our patient's symptoms have now resolved. It was not recorded in the 37 cases reported by Harms and Melcher. ${ }^{8}$
Atlanto-occipital posterior instrumented fusion, as described in this paper, for traumatic atlanto-occipital disruption has provided stability whilst preserving motion. Although technically demanding, there are significant advantages conferred over other techniques. A successful fusion was confirmed at radiological follow-up one year later. We would advocate its use for these injuries.

\section{Supplementary Material}

$\dddot{\mathrm{e}}$ A further opinion by Mr Brad Williamson is available with the electronic version of this article on our website at www.jbjs.org.uk

No benefits in any form have been received or will be received from a commercial party related directly or indirectly to the subject of this article.

\section{References}

1. Hadley M. Diagnois and management of traumatic atlanto-occipital dislocation injuries. Neurosurgery2002;50(Supp):105-13.

2. Sutterlin CE, Bianchi JR, Kunz DN, et al. Biomechanical evaluation of occipitocervical fixation devices. J Spinal Disord 2001;14:185-92.

3. Wertheim SB, BohIman HH. Occipitocervical fusion: indications, techniques, and longterm results in thirteen patients. J Bone Joint Surg [Am] 1987;69-A:833-6.

4. Oda I, Abumi K, Sell LC, et al. Biomechanical evaluation of five different occipitoatlanto-axial fixation techniques. Spine 1999;24:2377-82

5. Junge A, Krueger A, Petermann J, Gutzen J. Posterior atlanto-occipital dislocation and concomitant discoligamentous C3-C4 instability with survival. Spine 2001;26:1722-5.

6. Grob D. Transarticular screw fixation for atlanto-occipital dislocation. Spine 2001;26:7037 .

7. Sponseller PD, Cass JR. Atlanto-occipital fusion for dislocation in children with neurologic preservation: a case report. Spine 1997;22:344-7.

8. Harms J, Melcher RP. Posterior C1-C2 fusion with polyaxial screw and rod fixation. Spine 2001;26:2467-71.

9. Rumi MN, Kowalski JM, Gelb DE, Ludwig SC. Surgical stabilization for traumatic occipitocervical injuries. Current Opinion in Orthopedics 2002;13:232-7.

10. Traynelis VC, Marano GD, Dunker RO, Kaufman HH. Traumatic atlanto-occipital dislocation: case report. J Neurosurg 1986;65:863-70.

11. Anderson PA, Montesano PX. Morphology and treatment of occipital condyle fractures. Spine 1988;13:731-6.

12. Chattar-Cora D, Valenziano CP. Atlanto-occipital dislocation: a report of three patients and a review. J Orthop Trauma 2000;14:370-5.

13. Govender S, Vlok GJ, Fisher-Jeffes N, Du Preez CP. Traumatic dislocation of the atlanto-occipital joint. J Bone Joint Surg [Br]2003;85-B:875-8.

14. Powers B, Miller MD, Kramer RS, Martinez S, Gehweiler JA Jr. Traumatic anterior atlanto-occipital dislocation. Neurosurgery 1979;4:12-17.

15. Magerl F, Seemann P-S. Stable posterior fusion of the atlas and axis by transarticular screw fixation. In: Kehr P, Weindner A, eds. Cervical spine I. New York: Springer-Verlag, 1986:322-7.

16. Zipnick RI, Merola AA, Gorup J, et al. Occipital morphology: an anatomic guide to internal fixation. Spine 1996;21:1719-24.

17. Naderi S, Usal C, Tural AN, et al. Morphologic and radiologic anatomy of the occipital bone. J Spinal Disord 2001:14:500-3. 